\title{
Equational Theorem Proving Modulo
}

\author{
Dohan $\operatorname{Kim}^{(\varpi)}$ (1) and Christopher Lynch(1) \\ Clarkson University, Potsdam, NY, USA \\ $\{$ dohkim, clynch\}@clarkson.edu
}

\begin{abstract}
Unlike other methods for theorem proving modulo with constrained clauses $[12,13]$, equational theorem proving modulo with constrained clauses along with its simplification techniques has not been well studied. We introduce a basic paramodulation calculus modulo equational theories $E$ satisfying certain properties of $E$ and present a new framework for equational theorem proving modulo $E$ with constrained clauses. We propose an inference rule called Generalized E-Parallel for constrained clauses, which makes our inference system completely basic, meaning that we do not need to allow any paramodulation in the constraint part of a constrained clause for refutational completeness. We present a saturation procedure for constrained clauses based on relative reducibility and show that our inference system including our contraction rules is refutationally complete.
\end{abstract}

\section{Introduction}

Equations occur frequently in many areas of mathematics, logics, and computer science. Equational theorem proving $[6,8,19,22]$ is, in general, concerned with proving mathematical or logical statements in first-order clause logic with equality. While resolution [24] has been successful for theorem proving for first-order clause logic without equality, it has some limitations to deal with the equality predicate. For example, when dealing with the equality predicate using resolution, one must add the congruence axioms explicitly for each predicate and function symbol in order to express the properties of equality $[8,22]$.

Paramodulation [23] is based on the replacement of equals by equals, in order to improve the efficiency of resolution in equational theorem proving. However, paramodulation, in general, often produces a large amount of unnecessary clauses, so the search space for a refutation expands very rapidly. Therefore, various improvements have been developed for paramodulation. For example, it was shown that the functional reflexivity equations used by the traditional paramodulation rule [23] are not needed, and paramodulation into variables does not need to be allowed (see [8]).

Basic paramodulation $[9,20]$ restricts paramodulation by forbidding paramodulation at (sub)terms introduced by substitutions from previous inference steps, and uses orderings on terms and literals in order to further restrict paramodulation inferences. In $[21,26]$, basic paramodulation had been extended to basic paramodulation modulo associativity and commutativity $(A C)$ axioms.

(C) The Author(s) 2021

A. Platzer and G. Sutcliffe (Eds.): CADE 2021, LNAI 12699, pp. 166-182, 2021.

https://doi.org/10.1007/978-3-030-79876-5_10 
(See [25] also for basic paramodulation modulo the associativity $(A)$ axiom.) Basic paramodulation modulo $A C$ uses the symbolic constraints, overcoming a drawback of traditional paramodulation modulo $A C$ (see $[7,27]$ ) that often generates many slightly different permuted variants of clauses. For example, more than a million conclusions can possibly be generated by paramodulating the equation $x+x+x=x$ into the clause $P\left(y_{1}+y_{2}+y_{3}+y_{4}\right)$ for which + is an $A C$ symbol, since a minimal complete set of $A C$-unifiers for $x+x+x$ and $y_{1}+y_{2}+y_{3}+y_{4}$ contains more than a million $A C$-unifiers [21,26]. On the other hand, one only needs a single conclusion $P(x) \| x+x+x \approx_{A C}^{?} y_{1}+y_{2}+y_{3}+y_{4}$ for the above inference using basic paramodulation modulo $A C$ with an equality constraint.

In this paper, we present a new basic paramodulation calculus modulo equational theories $E$ (including $E=A C$ ) parameterized by a suitable $E$-compatible ordering $\succ$. Our main inference rule for basic paramodulation modulo $E$ is given (roughly) as follows:

$$
\frac{C \vee s \approx t\left\|\phi_{1} \quad D \vee L\left[s^{\prime}\right]\right\| \phi_{2}}{C \vee D \vee L[t] \| s \approx_{E}^{?} s^{\prime} \wedge \phi_{1} \wedge \phi_{2}}
$$

The equality constraints are inherited and the accumulated $E$-unification problems are kept in the constraint part of conclusion. Instead of generating as many conclusions as minimal and complete $E$-unifiers of two terms $s$ and $s^{\prime}$, a single conclusion is generated with its constraint keeping the $E$-unification problem of $s$ and $s^{\prime}$. Another key inference rule in our basic paramodulation calculus modulo $E$ is the Generalized $E$-Parallel (or $E$-Parallel) rule, adapted from our recent work on basic narrowing modulo [18]. This rule allows our basic paramodulation calculus to adapt the free case (i.e. $E=\emptyset$ ) to the modulo $E$ case (i.e. $E \neq \emptyset) .{ }^{1}$ For example, suppose that we have three clauses $1: a+b \approx c$, $2: a+(b+x) \approx c+x$, and $3:(a+a)+(b+b) \not z c+c$, where + is an $A C$ symbol with $+\succ a \succ b \succ c$. We use the $E$-Parallel rule from clause 1 and 2 and obtain the clause $4: a+(b+(a+b)) \approx c+c$, which derives a contradiction with clause 3 because $a+(b+(a+b)) \approx_{A C}(a+a)+(b+b)$ (i.e. the equality constraint is satisfiable). The details of this inference rule are discussed in Section 4.

Throughout this paper, we assume that (i) we are given an $E$-compatible reduction ordering $\succ$ on terms with the subterm property that is $E$-total on ground terms, (ii) $E$ has a finitary and complete unification algorithm, and (iii) $E$-congruence classes are finite. (If $E$ satisfies condition (i), then $E$ is necessarily regular [2].) With these assumptions of $E$, we can deal uniformly with different equational theories $E$ in our framework and show that our inference system including our contraction rules is refutationally complete.

The known practical theories satisfying the above assumptions of $E$ are $A C$ and finite permutation theories $[1,17]$. (For example, if one considers an $A C I$ symbol + using our approach, then $A C$ should be a modulo $E$ part and the idempotency axiom $(I: x+x \approx x)$ should be a part of the input formulas.) Although associative $(A)$-unification is infinitary, our approach is also applicable

${ }^{1}$ If $E=\emptyset$, then we may disregard the Generalized $E$-Parallel (or $E$-Parallel) rule along with the $E$-Completion rule and replace $E$-unification with syntactic unification. 
to the case where $E=A$ in practice, since there is a tool for $A$-unification which is guaranteed to terminate with a finite and complete set of $A$-unifiers for a significantly large class of $A$-unification problems (see [14]).

The longer version of this paper is found in [16].

\section{Preliminaries}

We assume that the reader has some familiarity with rewrite systems [3] (including the extended rewrite system for $R$ modulo $E$ (i.e. $R, E$ ) $[11,15]$ ) and unification [4]. We use the standard terminology of paramodulation $[6,9,22]$.

We denote by $T(\mathcal{F}, \mathcal{X})$ the set of terms over a finite set of function symbols $\mathcal{F}$ and a denumerable set of variables $\mathcal{X}$. An equation is an expression $s \approx t$, where $s$ and $t$ are (first-order) terms built from $T(\mathcal{F}, \mathcal{X})$. A literal is either an equation $L$ (a positive literal) or a negative equation $\neg L$ (a negative literal). A clause is a finite multiset of literals, written as a disjunction of literals $\neg A_{1} \vee \cdots \vee \neg A_{m} \vee B_{1} \vee \cdots \vee B_{n}$ or as an implication $\Gamma \rightarrow \Delta$, where the multiset $\Gamma$ is called the antecedent and the multiset $\Delta$ is called the succedent of the clause. (Recall that a multiset is an unordered collection with possible duplicate elements.)

An equational theory is a set of equations. (In this paper, an equational theory and a set of axioms are used interchangeably.) We denote by $\approx_{E}$ the least congruence on $T(\mathcal{F}, \mathcal{X})$ that is closed under substitutions and contains a set of equations $E$. If $s \approx_{E} t$ for two terms $s$ and $t$, then $s$ and $t$ are E-equivalent.

A (strict) ordering $\succ$ on terms is monotonic if $s \succ t$ implies $u[s]_{p} \succ u[t]_{p}$ for all $s, t, u$ and positions $p$. An ordering $\succ$ on terms is stable under substitutions if $s \succ t$ implies $s \sigma \succ t \sigma$ for all $s, t$, and substitutions $\sigma$. An ordering $\succ$ on terms is a rewrite ordering if it is monotonic and stable under substitutions. A well-founded rewrite ordering is a reduction ordering. An ordering $\succ$ on terms has the subterm property if $t[s]_{p} \succ s$ for all $s, t$, and $p \neq \lambda$. (In this paper, $\lambda$ denotes the top position.) A simplification ordering is a rewrite ordering with the subterm property. An ordering $\succ$ on terms is $E$-compatible if $s \succ t, s \approx_{E} s^{\prime}$, and $t \approx_{E} t^{\prime}$ implies $s^{\prime} \succ t^{\prime}$ for all $s, s^{\prime}, t$ and $t^{\prime}$. An ordering $\succ$ on ground terms is E-total if $s \not{ }_{E} t$ implies $s \succ t$ or $t \succ s$ for all ground terms $s$ and $t$.

Given a multiset $S$ and an $E$-compatible ordering $\succ$ on $S$, we say that $x$ is maximal (resp. strictly maximal) in $S$ if there is no $y \in S$ (resp. $y \in S \backslash\{x\}$ ) with $y \succ x$ (resp. $y \succeq x)$.

Clauses may also be considered as multisets of occurrences of equations. An occurrence of an equation $s \approx t$ in the antecedent of a clause is the multiset $\{\{s, t\}\}$, and in the succedent it is the multiset $\{\{s\},\{t\}\}$. We denote ambiguously all those orderings on terms, equations and clauses by $\succ$.

An equational theory is permutative if each equation in the theory contains the same symbols on both sides with the same number of occurrences. The depth of a term $t$ is defined as depth $(t)=0$ if $t$ is a variable or a constant and $\operatorname{depth}\left(f\left(s_{1}, \ldots, s_{n}\right)\right)=1+\max \left\{\operatorname{depth}\left(s_{i}\right) \mid 1 \leq i \leq n\right\}$. We say that an equational theory has maximum depth at most $k$ if the maximum depth of all terms in the 
equations in the theory is less than or equal to $k$.

A (Herbrand) interpretation $I$ is a congruence on ground terms. I satisfies (is a model of) a ground clause $\Gamma \rightarrow \Delta$, denoted by $I \models \Gamma \rightarrow \Delta$, if $I \nsupseteq \Gamma$ or $I \cap \Delta \neq \emptyset$. In this case, we say that $\Gamma \rightarrow \Delta$ is true in $I$. A ground clause $C$ follows from a set of ground clauses $\left\{C_{1}, \ldots, C_{k}\right\}=C$ if $C$ is true in every model of $\left\{C_{1}, \ldots, C_{k}\right\}$.

\section{Constrained Clauses}

Definition 1 (Constrained clauses) $[22,26]$ A constrained clause is a pair $C \| \phi$, where $C$ is a clause and $\phi$ is an equality constraint consisting of a conjunction of the form $s \approx_{E}^{?} t$ for terms $s$ and $t$. The set of solutions of a constraint $\phi$, denoted by $\operatorname{Sol}(\phi)$, is the set of the ground substitutions defined inductively as:

$$
\begin{gathered}
\operatorname{Sol}\left(\phi_{1} \wedge \phi_{2}\right)=\operatorname{Sol}\left(\phi_{1}\right) \cap \operatorname{Sol}\left(\phi_{2}\right), \\
\operatorname{Sol}\left(s \approx_{E}^{?} t\right)=\{\sigma \mid s \sigma \text { and } t \sigma \text { are } E \text {-equivalent }\}
\end{gathered}
$$

A constraint $\phi$ is satisfiable if it admits at least one solution.

A constrained clause with an unsatisfiable constraint is a tautology. If every ground substitution with domain $\operatorname{Vars}(\phi)$ of $C \| \phi$ is a solution of $\phi$, then $\phi$ is a tautological constraint. An unconstrained clause can also be considered as a constrained clause with a tautological constraint.

The main technical difficulties in lifting a reduced ground inference to an inference at the clause level in a basic paramodulation inference system involve a ground clause of the form $C \sigma:=D \sigma \vee x \sigma \approx t \sigma$ with $C:=D \vee x \approx t \| \phi$ and $\sigma \in \operatorname{Sol}(\phi)$, where $x \sigma \Rightarrow t \sigma \in R$ for a given ground rewrite system $R$. This motivates the following definition of irreducibility to lift a reduced ground inference to an inference at the clause level in our inference system. (See [9] also for order-irreducibility in the free case.)

Definition 2 (Order-irreducibility) Given a ground rewrite system $R$ and an equational theory $E$, a ground literal $L\left[l^{\prime}\right]_{p}$ is order-reducible (at position $p$ ) by $R, E$ with $l \Rightarrow r \in R$ if $l^{\prime} \approx_{E} l, l \succ r$ and $L \succ l \approx r$. A literal $L[s]$ is order-irreducible in $s$ by $R, E$ if $L[s]$ is not order-reducible at any position of $s$.

In Definition 2, the condition $L \succ l \approx r$ is always true when $L$ is a negative literal or else $l^{\prime}$ does not occur at the top (i.e. $p=\lambda$ ) of the largest term of $L$.

Definition 3 (Reduced ground instances) Given a ground rewrite system $R$ and an equational theory $E, C \sigma$ is a ground instance of $C \| \phi$ if $\sigma$ is a solution of $\phi$ (i.e. $\sigma \in \operatorname{Sol}(\phi))$. It is a reduced ground instance of $C \| \phi$ w.r.t. $R, E$ if $\sigma$ is a solution of $\phi$ and each ground literal $L[x \sigma]$ in $C \sigma$ is order-irreducible in $x \sigma$ by $R, E$ for each variable $x \in \operatorname{Vars}(C)$. In this case, $\sigma$ is a reduced solution of $C \| \phi$ w.r.t. $R, E$.

Definition 4 (A model of a constrained clause) An interpretation I satisfies (is a model of) a constrained clause $C \| \phi$, denoted by $I \models C \| \phi$, if it satisfies every ground instance of $C \| \phi$ (i.e. every $C \sigma$ for which $\sigma$ is a solution of $\phi$ ). 
Definition 5 (Reductiveness, weak reductiveness, semi-reductiveness, and weak maximality) An equation $s \approx t$ is reductive (resp. weakly reductive) for $C \| \phi:=$ $D \vee s \approx t \| \phi$ if there exists a ground instance $C \sigma$ such that $s \sigma \approx t \sigma$ is strictly maximal (resp. maximal) in $C \sigma$ with $s \sigma \succ t \sigma$. The clause $C \| \phi$ is simply called reductive if there exists a reductive equation $s \approx t$ for $C \| \phi$. A negative equation $u \not \approx v$ is semi-reductive (resp. weakly reductive) for $C\|\phi:=D \vee u \not \approx v\| \phi$ if there exists a ground instance $C \sigma$ such that $u \sigma \succ v \sigma$ (resp. $u \sigma \succ v \sigma$ and $u \sigma \not z v \sigma$ is maximal in $C \sigma$ ). A literal $L$ is weakly maximal for $C\|\phi:=D \vee L\| \phi$ if there exists a ground instance $C \sigma$ such that $L \sigma$ is maximal in $C \sigma$.

\section{Inference Rules}

The inference rules in our inference system are parameterized by a selection function $\mathcal{S}$ and an $E$-compatible reduction ordering $\succ$ with the subterm property that is $E$-total on ground terms, where $\mathcal{S}$ selects at most one (occurrence of a) negative literal in the clause part $C$ of each (constrained) clause $C \| \phi$. For technical convenience, if a literal $L$ is selected in $C$, then we also say that $L$ is selected in $C \| \phi$. In our inference rules, a literal in a clause $C \| \phi$ is involved in some inference if it is selected in $C$ (by $\mathcal{S}$ ) or nothing is selected and it is maximal in $C$ (cf. [8]). The following Basic Paramodulation rule is our main inference rule for equational theorem proving modulo $E$, where only the maximal sides of literals in clauses are involved in inferences by this rule. We rename variables in the premises in our inference rules if necessary so that no variable is shared between premises (i.e. standardized apart).

\section{Basic Paramodulation}

$$
\frac{C \vee s \approx t\left\|\phi_{1} \quad D \vee L\left[s^{\prime}\right]\right\| \phi_{2}}{C \vee D \vee L[t] \| s \approx_{E}^{?} s^{\prime} \wedge \phi_{1} \wedge \phi_{2}} \quad \text { if }
$$

1. $s^{\prime}$ is not a variable,

2. $s \approx t$ is reductive for the left premise, and $C$ contains no selected literal,

3. either one of the following three conditions is met:

(a) $L$ is selected in the right premise, and

$L$ is of the form $u\left[s^{\prime}\right] \not \approx v$ and is semi-reductive for the right premise.

(b) nothing is selected in the right premise, and

$L$ is of the form $u\left[s^{\prime}\right] \approx v$ and is reductive for the right premise.

(c) nothing is selected in the right premise, and

$L$ is of the form $u\left[s^{\prime}\right] \not \approx$ and is weakly reductive for the right premise.

\section{Equality Resolution}

$$
\frac{C \vee s \not t t \| \phi}{C \| s \approx_{E}^{?} t \wedge \phi} \quad \text { if }
$$


$s \not \approx t$ is selected, or else nothing is selected and $s \not \approx t$ is weakly maximal for the premise.

\section{E-Factoring}

$$
\frac{C \vee s \approx t \vee s^{\prime} \approx t^{\prime} \| \phi}{C \vee t \not t^{\prime} \vee s^{\prime} \approx t^{\prime} \| s \approx_{E}^{?} s^{\prime} \wedge \phi} \quad \text { if }
$$

$s \approx t$ is weakly reductive for the premise, and $C$ contains no selected literal.

\section{E-Completion}

$$
\frac{C \vee s \approx t \| \phi}{C \vee e_{1}[t]_{p} \approx e_{2} \| s \approx_{E}^{?} s^{\prime} \wedge \phi} \quad \text { if }
$$

1. $e_{1}\left[s^{\prime}\right]_{p} \approx e_{2} \in E$ and $p \neq \lambda$, where $s^{\prime}$ is not a variable,

2. $s \approx t$ is reductive for the premise, and $C$ contains no selected literal.

The above E-Completion rule is an adaptation of the E-closure [27] rule using equality constraints (cf. E-extension [5]).

\section{E-Parallel}

$$
\frac{C \vee s \approx t\left\|\phi_{1} \quad D \vee l \approx r\right\| \phi_{2}}{C \vee D \sigma \vee l \sigma \approx r \theta \| \phi_{1} \wedge \phi_{2}} \quad \text { if }
$$

1. $s \approx t$ is reductive for the left premise, and $C$ contains no selected literal,

2. $l \approx r$ is reductive for the right premise, and $D$ contains no selected literal,

3. both $l$ and $s$ are not variables,

4. $\sigma=\{x \mapsto s\}$ and $\theta=\{x \mapsto t\}$ for some variable $x \in \operatorname{Vars}(l) \cap \operatorname{Vars}(r)$ with $x \notin \operatorname{Vars}\left(\phi_{2}\right)$,

5. there is a term $u^{\prime}$ with $u^{\prime} \approx_{E} l \sigma$, such that $u^{\prime}$ is $R, E$-reducible with $R=\{l \Rightarrow r, s \Rightarrow t\}$ only at the top position (i.e. no strict subterm of $u^{\prime}$ is $R, E$-reducible).

\section{Generalized E-Parallel \\ $\frac{C \vee s \approx t\left\|\phi_{1} \quad D \vee l \approx r\right\| \phi_{2}}{C \vee D \sigma \vee l \sigma \approx r \theta \| \phi_{1} \wedge \phi_{2}} \quad$ if}

1. $s \approx t$ is reductive for the left premise, and $C$ contains no selected literal,

2. $l \approx r$ is reductive for the right premise, and $D$ contains no selected literal,

3. both $l$ and $s$ are not variables,

4. $e_{1}[u] \approx e_{2} \in E$, where $u$ is not a variable,

5. $\sigma=\left\{x \mapsto u[s]_{p}\right\}$ and $\theta=\left\{x \mapsto u[t]_{p}\right\}$ for some variable $x \in \operatorname{Vars}(l) \cap$ $\operatorname{Vars}(r)$ with $x \notin \operatorname{Vars}\left(\phi_{2}\right)$ and some position $p$, 
6. there is a term $u^{\prime}$ with $u^{\prime} \approx_{E} l \sigma$, such that $u^{\prime}$ is $R, E$-reducible with $R=\{l \Rightarrow r, s \Rightarrow t\}$ only at the top position.

We mark each clause produced by the Generalized E-Parallel (or E-Parallel) rule as "protected" so that it is protected from our contraction rules discussed in Section 5. (We simply say each marked clause is a protected clause.) Protected clauses behave the same way as other clauses in our inference rules, but our contraction rules are not applied to protected clauses (see Section 5 for details).

We may also use predicate terms $[6] P\left(t_{1}, \ldots, t_{n}\right)$ in our inference system, where a predicate term cannot be a proper subterm of any term. Note that a predicate term $P\left(t_{1}, \ldots, t_{n}\right)$ can be expressed as an equation $P\left(t_{1}, \ldots, t_{n}\right) \approx \top$, where $T$ is a special constant symbol minimal in the ordering $\succ$ and $P$ is considered as a function symbol. (In this sense, $\neg P\left(t_{1}, \ldots, t_{n}\right)$ can be expressed as $P\left(t_{1}, \ldots, t_{n}\right) \not \tau$.) In the remainder of this paper, by $\mathcal{B P}$ we denote the inference system consisting of the Basic Paramodulation, Equality Resolution, $E$-Factoring, E-Completion, and the Generalized $E$-Parallel rule. If $E$ is a permutative theory with maximum depth at most 2 (e.g. $E=A, C$, or $A C$ ), then we use the simpler $E$-Parallel rule instead of the Generalized $E$-Parallel rule in $\mathcal{B P}$ (see Lemma 6).

Example 1. Let + be an $A C$ symbol (in infix notation) with $+\succ a \succ b \succ 0$ and consider the following inconsistent set of clauses $1: x+0 \approx x, 2: a+a \approx 0,3$ : $b+b \approx 0$, and $4:(a+b)+(a+b) \not \approx 0$. Now we show how the empty clause (with a satisfiable constraint) is derived:

$5:(x+y)+z \approx x+0 \| y+z \approx{ }_{A C}^{?} a+a$ (E-Completion with 2 using the associativity axiom $x+(y+z) \approx(x+y)+z$.)

$6:((b+b)+y)+z \approx 0+0 \| y+z \approx_{A C}^{?} a+a$ (E-Parallel with 3 into 5 . In condition 5 of the $E$-Parallel rule, term $u^{\prime}$ corresponds to $(b+y)+(b+z)$ here. $)$ $7: 0+0 \not 00 \|((b+b)+y)+z \approx_{A C}^{?}(a+b)+(a+b) \wedge y+z \approx_{A C}^{?} a+a$ (Basic Paramodulation with 6 into 4$)$

$8: x \not \approx 0 \| x+0 \approx_{A C}^{?} 0+0 \wedge((b+b)+y)+z \approx_{A C}^{?}(a+b)+(a+b) \wedge y+z \approx_{A C}^{?} a+a$ (Basic Paramodulation with 1 into 7$)$

$9: \square \| x \approx_{A C}^{?} 0 \wedge x+0 \approx_{A C}^{?} 0+0 \wedge((b+b)+y)+z \approx_{A C}^{?}(a+b)+(a+b) \wedge y+z \approx_{A C}^{?}$ $a+a$ (Equality Resolution on 8)

In contrast, the existing approaches for basic paramodulation modulo $A C[21$, 26] use clauses 2 and 4, for example, and produce clause $5^{\prime}: 0+x \not \approx 0 \| x \approx_{A C}^{?} b+b$ and then clause $6^{\prime}: 0+y \not 0 \| x \approx_{A C}^{?} b+b \wedge y \approx_{A C}^{?} 0$ by their inference rules. Then $6^{\prime}$ is used to derive a contradiction with 1 . It can be viewed that $6^{\prime}$ is obtained from $5^{\prime}$ by an indirect paramodulation with 3 in the constraint part. In our approach, we simply block clauses like $5^{\prime}$ from further inferences (see Definition 12), and no direct or indirect paramodulation is allowed in the constraint part of any clause.

Example 2. Consider $S=\{f(g(x)) \approx x, a \approx b, c \not \approx g(b)\}$ and $E=\{f(g(g(a))) \approx$ c) with $f \succ g \succ a \succ b$, where $E$ is a regular theory with maximum depth 3 . The Generalized E-Parallel rule with premises $f(g(x)) \approx x$ and $a \approx b$ produces 
the conclusion $f(g(g(a))) \approx g(b)$. (Choose $l$ as $f(g(x)), s$ as $a$, and $u$ as $g(a)$ in the Generalized $E$-Parallel rule.) Then it is used to derive a contradiction with clause $c \not z g(b)$ since $f(g(g(a))) \approx_{E} c$.

In the above example, a suitable $E$-compatible reduction ordering $\succ$ on ground terms is obtained in such a way that given two ground terms, we rewrite each occurrence of $c$ in each ground term into $f(g(g(a)))$ at the same position with (the occurrence of) $c$ and then use the standard lexicographic path ordering $[3,22]$ for comparing (rewritten) ground terms without any occurrence of $c$. Then we may compare terms with variables by considering ground substitutions and using this ordering on ground terms.

In what follows, by the Parallel rule we mean the E-Parallel or the Generalized E-Parallel rule. First, observe that we cannot derive a contradiction in both Examples 1 and 2 using inference rules in $\mathcal{B P}$ without the Parallel rule. The intuition behind the Parallel rule is that above all, a reductive ground clause corresponds to a reductive ground conditional rewrite rule [19] with positive and negative conditions. Therefore, roughly speaking, the premises of the Parallel rule are reductive conditional rewrite rules with positive and negative conditions. (The Parallel rule applies to only reductive clauses.) Now the conclusion of the Parallel rule combines two steps: (i) instantiating a "problematic" variable in a special and restricted way, and (ii) selectively rewriting an instantiated term if conditions are met. (Therefore, conditions $C$ is included in the conclusion.) A problematic variable is often determined by a built-in equational theory $E$. It is mostly a variable produced by an $E$-Completion inference (see Example 1) for $A C$ cases, which is the counterpart of an extension variable for $A C$-extension [7,27].

Observe that the Generalized $E$-Parallel rule is more general than the $E$ Parallel rule. If $p$ is always the top position for the Generalized E-Parallel rule, then they are equivalent. This is the case for permutative theories with maximum depth at most 2 (e.g. $E=A, C$, or $A C$ ).

Lemma 6 If $E$ is a permutative theory with maximum depth at most 2, then the E-Parallel rule and the Generalized E-Parallel rule are equivalent, i.e., they generate the same conclusion for the same input premises.

Note that the E-Completion and the Parallel rule are not always needed for every built-in equational theory $E$. The following example is a simple variant of the reachability problem [15] modulo a permutation theory $[1,17]$, where $\neg P(f(c, b, b, d, e))$ is the query from the initial configuration $P(f(a, b, c, d, e))$. We may view $E$ in the following example as all permutations of variables $x_{1}, x_{2}, x_{3}, x_{4}$, and $x_{5}$, since the symmetric group $S_{5}$ is generated by two cycles (12) and (12345).

Example 3. Let $E=\left\{f\left(x_{1}, x_{2}, x_{3}, x_{4}, x_{5}\right) \approx f\left(x_{2}, x_{1}, x_{3}, x_{4}, x_{5}\right), f\left(x_{1}, x_{2}, x_{3}, x_{4}\right.\right.$, $\left.\left.x_{5}\right) \approx f\left(x_{2}, x_{3}, x_{4}, x_{5}, x_{1}\right)\right\}$ with $P \succ f \succ a \succ b \succ c \succ d \succ e$ and consider the following set of clauses 1: $\neg P(f(c, b, b, d, e)), 2: P(f(a, b, c, d, e))$, and 3: $f(a, b, x, y, z) \approx f(b, b, x, y, z)$. Basic Paramodulation with 3 into 2 
yields clause 4: $P(f(b, b, x, y, z)) \| f(a, b, x, y, z) \approx_{E}^{?} f(a, b, c, d, e)$. By applying Basic Paramodulation with 1 and 4 (using $P(f(c, b, b, d, e)) \not \approx \top$ and $\left.P(f(b, b, x, y, z)) \approx \top \| f(a, b, x, y, z) \approx_{E}^{?} f(a, b, c, d, e)\right)$ and then applying Equality Resolution, we have clause $5: \square \| f(b, b, x, y, z) \approx_{E}^{?} f(c, b, b, d, e) \wedge$ $f(a, b, x, y, z) \approx_{E}^{?} f(a, b, c, d, e)$. The equality constraint in 5 is satisfiable and we have a contradiction. Note that clause 4 schematizes the set of ground clauses $\{P(f(b, b, c, d, e)), P(f(b, b, c, e, d)), P(f(b, b, d, c, e)), P(f(b, b, d, e, c)), P(f(b, b, e$, $c, d)), P(f(b, b, e, d, c))\}$.

\section{Redundancy Criteria and Contraction Techniques}

Definition 7 (Relative reducibility) Given an equational theory $E$, a ground instance $C \sigma_{1}$ of $C \| \phi_{1}$ is reduced relative to a ground instance $D \sigma_{2}$ of $D \| \phi_{2}$ if for any rewrite system $R, C \sigma_{1}$ is a reduced ground instance of $C \| \phi_{1}$ w.r.t. $R, E$ whenever $D \sigma_{2}$ is a reduced ground instance of $D \| \phi_{2}$ w.r.t. $R, E$.

In what follows, the relation $\unlhd$ on terms represents the subterm relation, i.e., $s \unlhd t$ if $s$ is a subterm of $t$. The relation $\sqsubseteq$ on sets of terms is defined as follows: $\left\{s_{1}, \ldots, s_{m}\right\} \sqsubseteq\left\{t_{1}, \ldots, t_{n}\right\}$ if for all $1 \leq i \leq m$, there is some $1 \leq j \leq n$ such that $s_{i} \unlhd t_{j}$, and $\emptyset \sqsubseteq X$ for any set of terms $X$. Given a clause $C \| \phi$, we denote by $\operatorname{Ran}\left(\left.\sigma\right|_{\operatorname{Vars}(C)}\right)$ for some $\sigma \in \operatorname{Sol}(\phi)$ the range of the restriction of $\sigma$ to the set of variables $\operatorname{Var} s(C)$ if $\operatorname{Vars}(C) \neq \emptyset$. If $C$ is a ground clause with a tautological constraint (e.g. the empty constraint), then we set $\operatorname{Ran}\left(\left.\sigma\right|_{\operatorname{Var}(C)}\right)=\emptyset$. (Note that any ground substitution is a solution of a tautological constraint.)

We say that a clause $C \| \phi$ is a clause with a succedent top variable [21] w.r.t. $\sigma \in \operatorname{Sol}(\phi)$ if there is a variable $x \in \operatorname{Var} s(C) \cap \operatorname{Vars}(\phi)$ only appearing in equations $x \approx t$ of the succedent of $C$ with $x \sigma \succ t \sigma$ for some $t$. The following lemma, which directly follows from Definition 7 , is a sufficient syntactic condition for $C \sigma_{1}$ being reduced relative to $D \sigma_{2}$ in Definition 7 if $D \| \phi_{2}$ is not a clause with a succedent top variable w.r.t. $\sigma_{2}$. If $D \| \phi_{2}$ is a clause with a succedent top variable $x$ w.r.t. some $\sigma_{2} \in \operatorname{Sol}\left(\phi_{2}\right)$, then one may (partially) instantiate $x$ in $D$ with $\sigma_{2}$ if possible, so that one may use the syntactic condition for checking whether $C \sigma_{1}$ is reduced relative to $D \sigma_{2}$ as in the following lemma.

Lemma 8 Given an equational theory $E$, a ground instance $C \sigma_{1}$ of $C \| \phi_{1}$ is reduced relative to a ground instance $D \sigma_{2}$ of $D \| \phi_{2}$ if $\operatorname{Ran}\left(\left.\sigma_{1}\right|_{\operatorname{Vars}(C)}\right) \sqsubseteq$ $\operatorname{Ran}\left(\left.\sigma_{2}\right|_{V a r s(D)}\right)$ and $D \| \phi_{2}$ is not a clause with a succedent top variable w.r.t. $\sigma_{2}$.

In what follows, we denote by $E^{\prec C}$ (resp. $R^{\prec C}$ ) the set of ground instances of equations in $E$ (resp. the set of ground rewrite rules in $R$ ) smaller than the ground clause $C$ (w.r.t. $\succ$ ), and by $S$ modulo $E$ a set of clauses $S$ with a built-in equational theory $E$.

Definition 9 (Redundancy) A clause $C \| \phi$ is redundant in $S$ modulo $E$ (w.r.t. relative reducibility) if for every ground instance $C \sigma$, there exist ground 
instances $C_{1} \sigma_{1}, \ldots, C_{k} \sigma_{k}$ of clauses $C_{1}\left\|\phi_{1}, \ldots, C_{k}\right\| \phi_{k}$ in $S$ reduced relative to $C \sigma$, such that $C \sigma \succ C_{i} \sigma_{i}, 1 \leq i \leq k$, and $\left\{C_{1} \sigma_{1}, \ldots, C_{k} \sigma_{k}\right\} \cup R^{\prec C \sigma \cup E^{\prec C \sigma} \models C \sigma}$ for any ground rewite system $R$ contained in $\succ$. (In this case, we also say that each $C \sigma$ is redundant in $S$ modulo $E$ (w.r.t. relative reducibility).)

Definition 10 (Basic $E$-simplification) An equation $l \approx r$ simplifies a clause $C \vee L\left[l^{\prime}\right]_{p} \| \phi$ into $C \vee L[r \rho]_{p} \| \phi$ if the following conditions are met:

(i) $p$ is a non-variable position;

(ii) there is a substitution $\rho$ such that $l \rho \approx_{E} l^{\prime}, L\left[l^{\prime}\right] \succ l \rho \approx r \rho, \operatorname{Vars}(l \rho) \supseteq$ $\operatorname{Vars}(r \rho), l \rho \succ r \rho$, and $C \vee L\left[l^{\prime}\right]_{p} \| \phi$ is neither protected nor a clause with a succedent top variable w.r.t. any $\sigma \in \operatorname{Sol}(\phi)$.

Lemma 11 If an equation $l \approx r$ simplifies a clause $C \vee L\left[l^{\prime}\right]_{p} \| \phi$ into $C \vee$ $L[r \rho]_{p} \| \phi$ as in Definition 10, then $C \vee L\left[l^{\prime}\right]_{p} \| \phi$ is redundant in $S$ modulo $E$, where $S=\left\{l \approx r, C \vee L[r \rho]_{p} \| \phi\right\}$.

The following definition extends the blocking rule in the free case (see [9]) to the modulo case, where a blocked clause does not contribute to finding a refutation during a theorem proving derivation w.r.t. $\mathcal{B P}$ (see Definition 16) starting with an initial set of unconstrained clauses.

Definition 12 (Basic $E$-blocking) A clause $C \| \phi$ is blocked in $S$ modulo $E$ if the following conditions are met:

(i) $C \| \phi$ is not a clause with a succedent top variable w.r.t. any $\tau \in \operatorname{Sol}(\phi)$;

(ii) there is a variable $x \in \operatorname{Vars}(C) \cap \operatorname{Vars}(\phi)$ such that for every $\sigma \in \operatorname{Sol}(\phi)$, there exist ground instances $C_{1} \sigma_{1}, \ldots, C_{k} \sigma_{k}$ of clauses $C_{1}\left\|\phi_{1}, \ldots, C_{k}\right\| \phi_{k}$ in $S$ reduced relative to $C \sigma$, such that $C \sigma \succ C_{i} \sigma_{i}, 1 \leq i \leq k$, and $\left\{C_{1} \sigma_{1}, \ldots, C_{k} \sigma_{k}\right\} \cup E^{\prec C \sigma} \models x \sigma \approx s$ with $x \sigma \succ s$ for some ground term $s$.

Definition 13 (Basic E-instance) A clause $C \| \phi$ is a basic E-instance in $S$ modulo $E$ if the following conditions are met:

(i) $C \| \phi$ is protected;

(ii) there is a protected clause $D \| \psi \in S$ such that for every ground instance $C \sigma$ (resp. $D \tau)$ of $C \| \phi$ (resp. $D \| \psi$ ), there is a ground instance $D \tau$ (resp. $C \sigma$ ) of $D \| \psi$ (resp. $C \| \phi$ ) such that they are reduced relative to each other with $C \sigma=D \tau$.

Observe that protected clauses are produced in a restricted way (e.g. see condition 5 in the E-Parallel rule) and if two protected clauses are the same up to variable renaming, then they are basic $E$-instances of each other and they do not need to be distinguished.

Definition 14 (Redundancy of an inference) An inference $\pi$ with conclusion $D \| \phi$ is redundant in $S$ modulo $E$ (w.r.t. relative reducibility) if $D \| \phi$ is blocked or a basic $E$-instance in $S$ modulo $E$, or for every ground instance $\pi \sigma$ with maximal premise $C$ and conclusion $D \sigma$, there exist ground instances $C_{1} \sigma_{1}, \ldots, C_{k} \sigma_{k}$ of clauses $C_{1}\left\|\phi_{1}, \ldots, C_{k}\right\| \phi_{k}$ in $S$ reduced relative to $D \sigma$, such that $C \succ C_{i} \sigma_{i}$, $1 \leq i \leq k$, and $\left\{C_{1} \sigma_{1}, \ldots, C_{k} \sigma_{k}\right\} \cup R^{\prec C} \cup E^{\prec C} \models D \sigma$ for any ground rewrite system $R$ contained in $\succ$. 
The following lemma immediately follows from Definition 9 and the observation that if $\left\{C_{1} \sigma_{1}, \ldots, C_{k} \sigma_{k}\right\} \cup E^{\prec C \sigma}=C \sigma$, then $\left\{C_{1} \sigma_{1}, \ldots, C_{k} \sigma_{k}\right\} \cup R^{\prec C \sigma} \cup$ $E^{\prec C \sigma} \models C \sigma$ for any ground rewite system $R$ contained in $\succ$, which serves as a sufficient condition for redundancy of clauses. Also, if an (unconstrained) clause $C$ properly subsumes an (unconstrained) clause $C^{\prime} \vee D$ in the classical sense, where $C$ and $C^{\prime}$ are the same up to variable renaming, then it is easy to see that $C^{\prime} \vee D$ is redundant in $\{C\}$ modulo $E$.

Lemma $15 A$ clause $C \| \phi$ is redundant in $S$ modulo $E$ if for every ground instance $C \sigma$, there exist ground instances $C_{1} \sigma_{1}, \ldots, C_{k} \sigma_{k}$ of clauses $C_{1} \| \phi_{1}, \ldots$, $C_{k} \| \phi_{k}$ in $S$ reduced relative to $C \sigma$, such that $C \sigma \succ C_{i} \sigma_{i}, 1 \leq i \leq k$, and $\left\{C_{1} \sigma_{1}, \ldots, C_{k} \sigma_{k}\right\} \cup E^{\prec C \sigma}=C \sigma$.

Definition 16 (Theorem proving derivation) A theorem proving derivation is a sequence of sets of clauses $S_{0}=S, S_{1}, \ldots$ such that:

(i) Deduction: $S_{i}=S_{i-1} \cup\{C \| \phi\}$ for some $C \| \phi$ if it can be deduced from premises in $S_{i-1}$ by applying an inference rule in $\mathcal{B P}$ or basic $E$-simplification. (ii) Deletion: $S_{i}=S_{i-1} \backslash\{D \| \psi\}$ for some $D \| \psi$ if it is not protected, and is redundant or blocked in $S_{i-1}$ modulo $E$.

The set $S_{\infty}$ of persistent clauses is defined as $\bigcup_{i}\left(\bigcap_{j>i} S_{j}\right)$, which is called the limit of the derivation. A theorem proving derivation $S_{0}, S_{1}, S_{2}, \ldots$ is fair [6] w.r.t. the inference system $\mathcal{B P}$ if every inference $\pi$ by $\mathcal{B P}$ with premises in $S_{\infty}$ is redundant in $\bigcup_{j} S_{j}$ modulo $E$.

Definition 17 (Saturation w.r.t. relative reducibility) Given an equational theory $E$, we say that $S$ modulo $E$ is saturated under $\mathcal{B P}$ w.r.t. relative reducibility if every inference by $\mathcal{B P}$ with premises in $S$ is redundant in $S$ modulo $E$.

In what follows, we say that a clause $C \| \phi$ is non-protected redundant (resp. non-protected blocked) in $S$ modulo $E$ if it is not protected and is redundant (resp. blocked) in $S$ modulo $E$. (If $C \| \phi$ is non-protected redundant in $S$ modulo $E$, then we also say that each ground instance $C \sigma$ of $C \| \phi$ is non-protected redundant in $S$ modulo $E$.)

Lemma 18 (i) If $S \subseteq S^{\prime}$, then any clause which is non-protected redundant or non-protected blocked in $S$ modulo $E$ is also non-protected redundant or nonprotected blocked in $S^{\prime}$ modulo $E$.

(ii) Let $S \subseteq S^{\prime}$ such that all clauses in $S^{\prime} \backslash S$ are non-protected redundant or non-protected blocked in $S^{\prime}$ modulo E. Then (ii.1) any clause which is nonprotected redundant or non-protected blocked in $S^{\prime}$ modulo E is also non-protected redundant or non-protected blocked in $S$ modulo $E$, and (ii.2) any inference which is redundant in $S^{\prime}$ modulo $E$ is also redundant in $S$ modulo $E$.

Lemma 19 Let $S_{0}, S_{1}, \ldots$ be a fair theorem proving derivation w.r.t. $\mathcal{B P}$ such that $S_{0}$ is a set of unconstrained clauses. Then $S_{\infty}$ modulo $E$ is saturated under $\mathcal{B P}$ w.r.t. relative reducibility. 
Proof. If $S_{\infty}$ contains the empty clause, then it is immediate that $S_{\infty}$ modulo $E$ is saturated under $\mathcal{B P}$ w.r.t. relative reducibility, so we assume that the empty clause is not in $S_{\infty}$.

If a clause $C \| \phi$ is deleted in a theorem proving derivation, then we see that it is non-protected redundant or non-protected blocked in some $S_{j}$ modulo $E$. It is also non-protected redundant or non-protected blocked in $\bigcup_{j} S_{j}$ modulo $E$ by Lemma 18(i). Similarly, every clause in $\bigcup_{j} S_{j} \backslash S_{\infty}$ is non-protected redundant or non-protected blocked in $\bigcup_{j} S_{j}$ modulo $E$.

Now by fairness of the derivation, every inference $\pi$ by $\mathcal{B P}$ with premises in $S_{\infty}$ is redundant in $\bigcup_{j} S_{j}$ modulo $E$. Then by Lemma 18(ii.2) and the above, $\pi$ is also redundant in $S_{\infty}$ modulo $E$. Thus, $S_{\infty}$ modulo $E$ is saturated under $\mathcal{B P}$ w.r.t. relative reducibility.

\section{Refutational Completeness}

The soundness of $\mathcal{B P}$ (w.r.t. a fair theorem proving derivation) is straightforward, i.e., $S_{i} \cup E \models S_{i+1} \cup E$ for all $i \geq 0$. If the empty clause is in some $S_{j}$, then $S_{0} \cup E$ is unsatisfiable by the soundness of $\mathcal{B P}$. The following theorem states that $\mathcal{B P}$ with our contraction rules (i.e. basic $E$-simplification and basic $E$-blocking) is refutationally complete. In order to prove the following theorem, we adapt a variant of model construction techniques [7-9,21,27]. In this section, we assume that the equality is the only predicate by expressing other predicates (i.e. predicate terms) as (predicate) equations as discussed in Section 4.

Theorem 20 Let $S_{0}, S_{1}, \ldots$ be a fair theorem proving derivation w.r.t. $\mathcal{B P}$ such that $S_{0}$ is a set of unconstrained clauses. Then $S_{0} \cup E$ is unsatisfiable if and only if the empty clause is in some $S_{j}$.

Definition 21 (Model construction) Let $S$ be a set of (constrained) clauses. We use induction on $\succ$ to define the sets Rules $C, R_{C}, E_{C}$, and $I_{C}$, for all ground instances $C$ of clauses in $S$. Let $C$ be such a ground instance of a clause in $S$ and suppose that Rules $C^{\prime}$ has been defined for all ground instances $C^{\prime}$ of clauses in $S$ for which $C \succ C^{\prime}$. Then we define by $R_{C}=\bigcup_{C \succ C^{\prime}}$ Rules $C^{\prime}$ and by $E_{C}$ the set of ground instances $e_{1} \approx e_{2}$ of equations in $E$, such that $C \succ e_{1} \approx e_{2}$, and $e_{1}$ and $e_{2}$ are both irreducible by $R_{C}$. We also define by $I_{C}$ the interpretation $\left(R_{C} \cup E_{C}\right)^{*}$ (i.e. the least congruence containing $\left.R_{C} \cup E_{C}\right)$.

Now let $C:=D \vee s \approx t$ be a reduced ground instance of a clause in $S$ w.r.t. $R_{C}$ such that $C$ is not an instance of a clause with a selected literal. Then $C$ produces the set of ground rewrite rules Rules $_{C}=\left\{u \Rightarrow t \mid u \approx_{E} s\right.$ and $u$ is irreducible by $\left.R_{C}\right\}$ if the following conditions are met: (1) $I_{C} \not \models C$ (resp. $I_{C} \not \models D$ ) if $C$ is an instance of a non-protected clause (resp. protected clause), (2) $I_{C} \not \forall t \approx t^{\prime}$ for every $s^{\prime} \approx t^{\prime}$ in $D$ with $s^{\prime} \approx_{E} s,(3) s \approx t$ is reductive for $C$, and (4) there exists $u$ with $u \approx_{E} s$ for which $u$ is irreducible by $R_{C}$. We say that $C$ is productive and produces Rules $_{C}$ if it satisfies all of the above conditions. Otherwise, Rules $_{C}=\emptyset$. Finally, we define $R_{S}=\bigcup_{C} R_{C}, E_{S}=\bigcup_{C} E_{C}$, and $I_{S}=\left(R_{S} \cup E_{S}\right)^{*}$. 
We may include the special non-productive ground clause $t t \approx t t$ in $S$ for the above (inductive) definition, where $t t \approx t t$ is assumed to be greater than all ground instances of clauses in $S \cup E$ w.r.t. $\succ$ other than $t t \approx t t$ itself (see [21,27]). (If $C$ is the strictly maximal ground instance among ground instances of clauses in $S$ and is productive, then $R_{S}$ may not include Rules $_{C}$ by the above inductive definition of $R_{C}$ without $t t \approx t t$.) In what follows, we say that a ground instance $\pi \sigma$ of an inference $\pi$ with premises in $S$ is reduced if each premise and conclusion of $\pi \sigma$ is a reduced ground instance of a clause in $S \cup E$ w.r.t. $R_{S}, E_{S}$.

Definition 22 (Redundancy w.r.t. $R_{S}, E_{S}$ ) A clause $C \| \phi$ is redundant in $S$ modulo $E$ w.r.t. $R_{S}, E_{S}$ if for every reduced ground instance $C \sigma$ w.r.t. $R_{S}, E_{S}$, there exist reduced ground instances $C_{1} \sigma_{1}, \ldots, C_{k} \sigma_{k}$ of clauses $C_{1}\left\|\phi_{1} \ldots C_{k}\right\| \phi_{k}$ in $S$ w.r.t. $R_{S}, E_{S}$, such that $C \sigma \succ C_{i} \sigma_{i}, 1 \leq i \leq k$, and $\left\{C_{1} \sigma_{1}, \ldots, C_{k} \sigma_{k}\right\} \cup$ $R_{S}^{\prec C \sigma} \cup E^{\prec C \sigma} \models C \sigma$. (In this case, we also say that each $C \sigma$ is redundant in $S$ modulo $E$ w.r.t. $R_{S}, E_{S}$.)

An inference $\pi$ with conclusion $D \| \phi$ is redundant in $S$ modulo $E$ w.r.t. $R_{S}, E_{S}$ if $D \| \phi$ is blocked or a basic $E$-instance in $S$ modulo $E$, or for every reduced ground instance $\pi \sigma$ with maximal premise $C$ and conclusion $D \sigma$, there exist reduced ground instances $C_{1} \sigma_{1}, \ldots, C_{k} \sigma_{k}$ of clauses $C_{1}\left\|\phi_{1}, \ldots, C_{k}\right\| \phi_{k}$ in $S$ w.r.t. $R_{S}, E_{S}$, such that $C \succ C_{i} \sigma_{i}, 1 \leq i \leq k$, and $\left\{C_{1} \sigma_{1}, \ldots, C_{k} \sigma_{k}\right\} \cup R_{S}^{\prec C} \cup$ $E^{\prec C}=D \sigma$.

Definition 23 (Saturation w.r.t. $R_{S}, E_{S}$ ) Given an equational theory $E$, we say that $S$ modulo $E$ is saturated under $\mathcal{B P}$ w.r.t. $R_{S}, E_{S}$ if every inference by $\mathcal{B P}$ with premises in $S$ is redundant in $S$ modulo $E$ w.r.t. $R_{S}, E_{S}$.

Lemma 24 (i) There are no overlaps among the left-hand sides of rules in $R_{S}$. (ii) A term $t$ is reducible by $R_{S}$ if and only if it is reducible by $R_{S}, E_{S}$ at the same position.

(iii) For every $l \Rightarrow r, s \Rightarrow t \in R_{S}$, if $l \approx_{E} s$, then $r$ and $t$ are the same term.

(iv) $R_{S} / E_{S}$ is terminating.

(v) For ground terms $u$ and $v$, if $I_{S} \models u \approx v$, then $u \downarrow_{R_{S}, E_{S}} v$.

(vi) If a ground instance $C \theta:=D \theta \vee l \theta \approx r \theta$ of a clause $C\|\phi:=D \vee l \approx r\| \phi$ is productive, then it is a reduced ground instance of $C \| \phi$ w.r.t. $R_{S}, E_{S}$.

The proofs of (i), (ii), and (iii) in Lemma 24 follow from the construction of $R_{S}$ in Definition 21. For (iv), since $R_{S}$ is contained in an $E$-compatible reduction ordering $\succ$ on terms that is $E$-total on ground terms, $R_{S} / E_{S}$ is terminating. Meanwhile, Lemma 24(v) describes the ground Church-Rosser property [19] of $R_{S}, E_{S}$. Since $R_{S} / E_{S}$ is terminating by (iv), this shows that $R_{S}, E_{S}$ is ground convergent modulo $E_{S}$. In the following, we assume that any saturated clause set under $\mathcal{B P}$ is obtained from an initial set of clauses without constraints.

Lemma 25 Let $S$ modulo $E$ be saturated under $\mathcal{B P}$ w.r.t. $R_{S}, E_{S}$ not containing the empty clause and let $C$ be a reduced ground instance of a clause in $S$ w.r.t. $R_{S}, E_{S}$ or a ground instance of an equation in $E$. Then $C$ is true in $I_{S}$. More specifically, 
(i) $C$ is not an instance of a blocked clause in $S$ modulo $E$.

(ii) If $C$ is redundant in $S$ modulo $E$ w.r.t. $R_{S}, E_{S}$, then it is true in $I_{S}$.

(iii) If $C$ is an instance of a clause with a selected literal, then it is true in $I_{S}$.

(iv) If $C$ contains a maximal negative literal (w.r.t. $\succ$ ) and is not an instance of a clause with a selected literal, then it is true in $I_{S}$.

(v) If $C$ is an instance of an equation in $E$, then it is true in $I_{S}$.

(vi) If $C$ is an instance of a protected clause or a basic E-instance of it, then it is true in $I_{S}$.

(vii) If $C$ is non-productive, then it is true in $I_{S}$.

(viii) If $C:=C^{\prime} \vee s \approx t$ is productive and produces Rules $C$ with $s \Rightarrow t \in$ Rules $_{C}$, then $C^{\prime}$ is false and $C$ is true in $I_{S}$.

We leave it to the reader to verify the following lemma using the definitions of redundancy of an inference w.r.t. relative reducibility and w.r.t. $R_{S}, E_{S}$, along with Lemma 19.

Lemma 26 Let $S_{0}, S_{1}, \ldots$ be a fair theorem proving derivation w.r.t. $\mathcal{B P}$ such that $S_{0}$ is a set of unconstrained clauses. Then $S_{\infty}$ modulo $E$ is saturated under $\mathcal{B P}$ w.r.t. $R_{S_{\infty}}, E_{S_{\infty}}$.

Theorem 27 Let $S_{0}, S_{1}, \ldots$ be a fair theorem proving derivation w.r.t. $\mathcal{B P}$ such that $S_{0}$ is a set of unconstrained clauses. If $S_{\infty}$ does not contain the empty clause, then $I_{S_{\infty}}=S_{0} \cup E$ (i.e., $S_{0} \cup E$ is satisfiable).

Proof. By Lemma 26, we know that $S_{\infty}$ modulo $E$ is saturated under $\mathcal{B P}$ w.r.t. $R_{S_{\infty}}, E_{S_{\infty}}$. Let $C$ be a ground instance of an equation in $E$ or a ground instance of a clause $C^{\prime}$ in $S_{0}$. By Lemma $25(\mathrm{v})$, if $C$ is a ground instance of an equation in $E$, then it is true in $I_{S_{\infty}}$. Therefore, we assume that $C$ is not a ground instance of an equation in $E$. Suppose first that $C:=C^{\prime} \sigma^{\prime}$ is a reduced ground instance of $C^{\prime} \in S_{0}$ w.r.t. $R_{S_{\infty}}, E_{S_{\infty}}$. Then there are two cases to consider. If $C^{\prime} \in S_{\infty}$, then $C$ is true in $I_{S_{\infty}}$ by Lemma 25. Otherwise, if $C^{\prime} \notin S_{\infty}$, then $C^{\prime}$ is (non-protected) redundant in some $S_{j}$ modulo $E$ w.r.t. relative reducibility because $C^{\prime} \in S_{0}$ (with the empty constraint) is neither protected nor can it be a blocked clause in some $S_{j}$ modulo $E$. Thus, $C^{\prime}$ is (nonprotected) redundant in $\bigcup_{j} S_{j}$ modulo $E$ w.r.t. relative reducibility, and hence is (non-protected) redundant in $S_{\infty}$ modulo $E$ w.r.t. relative reducibility by Lemma 18. It follows that there exist ground instances $C_{1} \sigma_{1}, \ldots, C_{k} \sigma_{k}$ of clauses $C_{1}\left\|\phi_{1}, \ldots, C_{k}\right\| \phi_{k}$ in $S_{\infty}$ reduced relative to $C$, such that $C \succ C_{i} \sigma_{i}, 1 \leq i \leq k$, and $\left\{C_{1} \sigma_{1}, \ldots, C_{k} \sigma_{k}\right\} \cup R^{\prec C} \cup E^{\prec C} \models C$ for any ground rewrite system $R$ contained in $\succ$. Since $C$ is a reduced ground instance of $C^{\prime}$ w.r.t. $R_{S_{\infty}}, E_{S_{\infty}}$, we see that $C_{i} \sigma_{i}, 1 \leq i \leq k$, are also reduced ground instances w.r.t. $R_{S_{\infty}}, E_{S_{\infty}}$ by Definition 7 and are true in $I_{S_{\infty}}$ by Lemma 25. Similarly, $R_{S_{\infty}}^{\prec C}$ and $E^{\prec C}$ are true in $I_{S_{\infty}}$ by Lemma 25, and hence we may infer that $C$ is also true in $I_{S_{\infty}}$.

Now suppose that $C:=C^{\prime} \sigma^{\prime}$ is a reducible ground instance of $C^{\prime} \in S_{0}$ w.r.t. $R_{S_{\infty}}, E_{S_{\infty}}$. Let $\sigma^{\prime \prime}$ be a ground substitution such that $x \sigma^{\prime \prime}=x \sigma^{\prime} \downarrow_{R_{S_{\infty}}, E_{S_{\infty}}}$ for each $x \in \operatorname{Vars}\left(C^{\prime}\right)$. Since $C^{\prime} \sigma^{\prime \prime}$ is a reduced ground instance of $C^{\prime} \in S_{0}$ w.r.t. $R_{S_{\infty}}, E_{S_{\infty}}, C^{\prime} \sigma^{\prime \prime}$ is true in $I_{S_{\infty}}$ by the previous paragraph, and hence $C$ is also true in $I_{S_{\infty}}$. 
We may now present the proof that $\mathcal{B P}$ with our contraction rules is refutationally complete.

Proof of Theorem 20 Let $S_{0}, S_{1}, \ldots$ be a fair theorem proving derivation w.r.t. $\mathcal{B P}$ such that $S_{0}$ is a set of unconstrained clauses. If the empty clause is in some $S_{j}$, then $S_{0} \cup E$ is unsatisfiable by the soundness of $\mathcal{B P}$. Otherwise, if the empty clause is not in $S_{k}$ for all $k$, then by the soundness of $\mathcal{B P}, S_{\infty}$ does not contain the empty clause, and hence $S_{0} \cup E$ is satisfiable by Theorem 27 .

\section{Conclusion}

We have presented a basic paramodulation calculus modulo and provided a framework for equational theorem proving modulo equational theories $E$ satisfying some properties of $E$ using constrained clauses, where a constrained clause may schematize a set of unconstrained clauses by keeping $E$-unification problems in its constraint part. Our results imply that we can deal uniformly with different equational theories $E$ in our equational theorem proving modulo framework. We only need a single refutational completeness proof for our basic paramodulation calculus modulo $E$ for different equational theories $E$.

Our contraction techniques (i.e. basic $E$-simplification and basic $E$-blocking) for constrained clauses can also be applied uniformly for different equational theories $E$ satisfying some properties of $E$ in our equational theorem proving modulo framework. Since a constrained clause may schematize a set of unconstrained clauses, the simplification or deletion of a constrained clause may correspond to the simplification or deletion of a set of unconstrained clauses. We have proposed a saturation procedure for constrained clauses based on relative reducibility and showed the refutational completeness of our inference system using a saturated clause set (w.r.t. $\succ$ ).

Some possible improvements remain to be done. One of the main issues is the broadening the scope of our equational theorem proving modulo $E$ to more equational theories $E$. This can be achieved by dropping or weakening some ordering requirements of $\succ$ (e.g. monotonicity of $\succ$ ) for a basic paramodulation calculus modulo $E$, while maintaining the refutational completeness of the calculus (cf. [10]). This can also be achieved by finding suitable $E$-compatible orderings for more equational theories $E$. In fact, we provided an $E$-compatible simplification ordering $\succ$ on terms that is $E$-total on ground terms for finite permutation theories $E$ in [17], which allows us to provide a refutationally complete equational theorem proving with built-in permutation theories using the results of this paper. Since permutations play an important role in mathematics and many fields of science including computer science, we believe that developing applications for equational theorem proving with built-in permutation theories is another promising future research direction. 


\section{References}

1. Avenhaus, J.: Efficient Algorithms for Computing Modulo Permutation Theories. In: Basin, D., Rusinowitch, M. (eds.) Automated Reasoning - IJCAR 2004, Cork, Ireland, July 4-8. pp. 415-429. Springer, Berlin, Heidelberg (2004)

2. Baader, F.: Combination of compatible reduction orderings that are total on ground terms. In: Winskel, G. (ed.) Proceedings of the Twelfth Annual IEEE Symposium on Logic in Computer Science. pp. 2-13. IEEE Computer Society Press, Warsaw, Poland (1997)

3. Baader, F., Nipkow, T.: Term Rewriting and All That. Cambridge University Press, Cambridge, UK (1998)

4. Baader, F., Snyder, W.: Unification Theory. In: Handbook of Automated Reasoning, chap. 8, pp. 445 - 532. Volume I, Elsevier, Amsterdam (2001)

5. Bachmair, L., Dershowitz, N.: Completion for rewriting modulo a congruence. Theoretical Computer Science 67(2), 173 - 201 (1989)

6. Bachmair, L., Ganzinger, H.: Rewrite-based Equational Theorem Proving with Selection and Simplification. J. Log. Comput. 4(3), 217-247 (1994)

7. Bachmair, L., Ganzinger, H.: Associative-commutative superposition. In: Dershowitz, N., Lindenstrauss, N. (eds.) Conditional and Typed Rewriting Systems. pp. 1-14. Springer, Berlin, Heidelberg (1995)

8. Bachmair, L., Ganzinger, H.: Equational Reasoning in Saturation-Based Theorem Proving. In: Bibel, W., Schmitt, P. (eds.) Automated Deduction. A basis for applications, chap. 11, p. 353-397. Volume I, Kluwer, Dordrecht, Netherlands (1998)

9. Bachmair, L., Ganzinger, H., Lynch, C., Snyder, W.: Basic Paramodulation. Information and Computation 121(2), 172 - 192 (1995)

10. Bofill, M., Rubio, A.: Paramodulation with Non-Monotonic Orderings and Simplification. Journal of Automated Reasoning 50, 51-98 (2013)

11. Dershowitz, N., Plaisted, D.A.: Rewriting. In: Handbook of Automated Reasoning, chap. 9, pp. 535 - 610. Volume I, Elsevier, Amsterdam (2001)

12. Dowek, G.: Polarized Resolution Modulo. In: Calude, C.S., Sassone, V. (eds.) Theoretical Computer Science. pp. 182-196. Springer, Berlin, Heidelberg (2010)

13. Dowek, G., Hardin, T., Kirchner, C.: Theorem Proving Modulo. Journal of Automated Reasoning 31(1), 33-72 (2003)

14. Durán, F., Eker, S., Escobar, S., Martí-Oliet, N., Meseguer, J., Talcott, C.: Associative Unification and Symbolic Reasoning Modulo Associativity in Maude. In: Rusu, V. (ed.) Rewriting Logic and Its Applications. pp. 98-114. Springer, Cham (2018)

15. Escobar, S., Sasse, R., Meseguer, J.: Folding variant narrowing and optimal variant termination. The Journal of Logic and Algebraic Programming 81(7), 898 - 928 (2012)

16. Kim, D., Lynch, C.: Equational Theorem Proving Modulo (2021), Technical Report, Web link: https://people.clarkson.edu/ clynch/PAPERS/etpm.pdf

17. Kim, D., Lynch, C.: An RPO-based ordering modulo permutation equations and its applications to rewrite systems. In: 6th International Conference on Formal Structures for Computation and Deduction, FSCD 2021, Buenos Aires, Argentina (Virtual Conference), July 17-24, to appear. vol. 195, pp. 19:1-19:17. LIPIcs (2021), preprint: http://people.clarkson.edu/ dohkim/tech_reports/ERPO.pdf

18. Kim, D., Lynch, C., Narendran, P.: Reviving Basic Narrowing Modulo. In: Herzig, A., Popescui, A. (eds.) Frontiers of Combining Systems. pp. 313-329. Springer, Cham, Switzerland (2019) 
19. Kirchner, C., Kirchner, H.: Rewriting, Solving, Proving (1999), Preliminary version: http://citeseerx.ist.psu.edu/viewdoc/summary?doi=10.1.1.144.5349

20. Nieuwenhuis, R., Rubio, A.: Basic superposition is complete. In: Krieg-Brückner, B. (ed.) ESOP '92. pp. 371-389. Springer, Berlin, Heidelberg (1992)

21. Nieuwenhuis, R., Rubio, A.: Paramodulation with Built-in AC-Theories and Symbolic Constraints. Journal of Symbolic Computation 23(1), 1 - 21 (1997)

22. Nieuwenhuis, R., Rubio, A.: Paramodulation-based theorem proving. In: Handbook of Automated Reasoning, chap. 7, pp. 371-443. Volume I, Elsevier, Amsterdam (2001)

23. Robinson, G., Wos, L.: Paramodulation and theorem-proving in first-order theories with equality. In: Meltzer, B., Michie, D. (eds.) Machine Intelligence 4, pp. 133-150. American Elsevier, New York (1969)

24. Robinson, J.A.: A machine-oriented logic based on the resolution principle. J. ACM 12(1), 23-41 (1965)

25. Rubio, A.: Theorem Proving modulo Associativity. In: Büning, H.K. (ed.) Computer Science Logic. pp. 452-467. Springer, Berlin, Heidelberg (1996)

26. Vigneron, L.: Associative-Commutative Deduction with Constraints. In: Bundy, A. (ed.) Automated Deduction - CADE-12. pp. 530-544. Springer, Berlin (1994)

27. Wertz, U.: First-order theorem proving modulo equations. Tech. Rep. MPI-I-92216, Max-Planck-Institut für Informatik, Saarbrücken (1992)

Open Access This chapter is licensed under the terms of the Creative Commons Attribution 4.0 International License (http://creativecommons.org/licenses/by/ 4.0/), which permits use, sharing, adaptation, distribution and reproduction in any medium or format, as long as you give appropriate credit to the original author(s) and the source, provide a link to the Creative Commons license and indicate if changes were made.

The images or other third party material in this chapter are included in the chapter's Creative Commons license, unless indicated otherwise in a credit line to the material. If material is not included in the chapter's Creative Commons license and your intended use is not permitted by statutory regulation or exceeds the permitted use, you will need to obtain permission directly from the copyright holder.

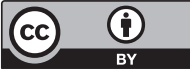

